\title{
Commissioning, characterization and operation of the dual-recycled GEO 600
}

\author{
J R Smith ${ }^{1}$, B Allen ${ }^{3}$, P Aufmuth ${ }^{1}$, C Aulbert ${ }^{3}$, S Babak ${ }^{4}$, \\ R Balasubramanian ${ }^{4}$, B W Barr ${ }^{2}$, S Berukoff ${ }^{3}$, A Bunkowski $^{1}$, \\ G Cagnoli ${ }^{2}$, C A Cantley ${ }^{2}$, M M Casey ${ }^{2}$, S Chelkowski $^{1}$, D Churches ${ }^{4}$, \\ T Cokelaer $^{4}$, C N Colacino ${ }^{1}$, D R M Crooks ${ }^{2}$, C Cutler $^{3}$, K Danzmann ${ }^{1}$, \\ R Davies ${ }^{4}$, R J Dupuis ${ }^{2}$, E Elliffe ${ }^{2}$, C Fallnich ${ }^{5}$, A Franzen ${ }^{1}$, A Freise ${ }^{1}$, \\ S Goßler ${ }^{1}$, A Grant ${ }^{2}$, H Grote ${ }^{1}$, S Grunewald ${ }^{3}$, J Harms ${ }^{1}$, G Heinzel ${ }^{1}$, \\ I S Heng ${ }^{1}$, A Hepstonstall ${ }^{2}$, M Heurs ${ }^{1}$, M Hewitson ${ }^{2}$, S Hild ${ }^{1}$, \\ J Hough $^{2}$, Y Itoh ${ }^{3}$, R Jones ${ }^{2}$, S H Huttner ${ }^{2}$, K Kawabe ${ }^{1}$, C Killow ${ }^{2}$, \\ K Kötter ${ }^{1}$, B Krishnan ${ }^{3}$, V Leonhardt ${ }^{1}$, H Lück ${ }^{1}$, B Machenschalk ${ }^{3}$, \\ M Malec $^{1}$, R A Mercer ${ }^{6}$, C Messenger $^{6}$, S Mohanty $^{3}$, K Mossavi $^{1}$, \\ S Mukherjee ${ }^{3}$, P Murray ${ }^{2}$, S Nagano ${ }^{1}$, G P Newton ${ }^{2}$, M A Papa ${ }^{1,3}$,

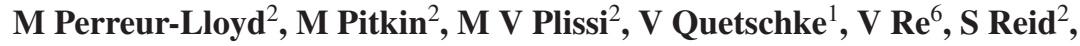 \\ L Ribichini ${ }^{1}$, D I Robertson ${ }^{2}$, N A Robertson ${ }^{2,8}$, J D Romano ${ }^{4}$, \\ S Rowan ${ }^{2}$, A Rüdiger ${ }^{1}$, B S Sathyaprakash ${ }^{4}$, R Schilling ${ }^{1}$, \\ R Schnabel ${ }^{1}$, B F Schutz ${ }^{3,4}$, F Seifert ${ }^{1}$, A M Sintes ${ }^{7}$, P H Sneddon ${ }^{2}$, \\ K A Strain ${ }^{2}$, I Taylor ${ }^{4}$, R Taylor ${ }^{2}$, A Thüring ${ }^{1}$, C Ungarelli ${ }^{6}$,

 \\ P Williams $^{3}$, B Willke ${ }^{1}$, W Winkler ${ }^{1}$, G Woan $^{2}$ and I Zawischa ${ }^{5}$

\footnotetext{
${ }^{1}$ Max-Planck-Institut für Gravitationsphysik (Albert-Einstein-Institut) und Universität Hannover, Callinstr 38, D-30167 Hannover, Germany

${ }^{2}$ Department of Physics \& Astronomy, University of Glasgow, Glasgow G12 8QQ, UK

${ }^{3}$ Max-Planck-Institut für Gravitationsphysik (Albert-Einstein-Institut), Golm Am Mühlenberg 1, 14476 Golm, Germany

${ }^{4}$ Department of Physics and Astronomy, Cardiff University, PO Box 913, Cardiff CF2 3YB, UK

${ }^{5}$ Laser Zentrum Hannover eV, Hollerithallee 8, 30419 Hannover, Germany

${ }^{6}$ School of Physics and Astronomy, The University of Birmingham, Edgbaston, Birmingham B15 2TT, UK

${ }^{7}$ Departament de Fisica, Universitat de les Illes Balears, E-07122 Palma de Mallorca, Spain

E-mail: joshua.smith@aei.mpg.de
} \\ ${ }^{8}$ Edward L Ginzton Laboratory, Stanford University, Stanford, CA 94305-4088, USA
}

Received 18 April 2004

Published 28 September 2004

Online at stacks.iop.org/CQG/21/S1737

doi:10.1088/0264-9381/21/20/016

\section{Abstract}

The German-British laser-interferometric gravitational-wave detector GEO 600 is currently being commissioned as part of a worldwide network of gravitational-wave detectors. GEO 600 recently became the first kilometrescale interferometer to employ dual recycling - an optical configuration that combines power recycling and signal recycling. We present a brief overview 
of the commissioning of this dual-recycled interferometer, the performance results achieved during a subsequent extended data-taking period, and the plans intended to bring GEO 600 to its final configuration.

PACS numbers: $04.80 . \mathrm{Nn}, 95.55 . \mathrm{Ym}$, 95.75.Kk

(Some figures in this article are in colour only in the electronic version)

\section{Introduction}

The first generation of an international network of earth-bound kilometre-scale laserinterferometric gravitational-wave detectors is currently moving from the commissioning phase to the long-term data-taking phase. These detectors are designed to search for gravitational waves emitted from a number of different astrophysical sources including supernovae explosions, non-symmetric spinning neutron stars, inspiralling binary systems of neutron stars and/or black holes and gravitational background radiation originating from the big bang. In addition to these potential sources, unknown sources may produce gravitational radiation of detectable strength. A summary of the current understanding of gravitational-wave sources and event rates is given in [1].

The German-British GEO 600 detector with $600 \mathrm{~m}$ arm length is one member of this network. It is joined by the three detectors (two with $4 \mathrm{~km}$ arm length and one with $2 \mathrm{~km}$ arm length) of the USA-based LIGO project [2], the Japanese TAMA detector with $300 \mathrm{~m}$ arm length [3] and the French-Italian VIRGO detector with $3 \mathrm{~km}$ arm length [4].

GEO 600 recently became the first of these detectors to employ dual recycling-an advanced optical configuration combining power recycling and signal recycling [5-8]. This, and other novel techniques already in use at GEO 600, such as triple pendulums including a quasi-monolithic lower stage made of fused silica $[9,10]$, thermal correction of the radii of curvature of mirrors [11], two sequential suspended mode-cleaners [12] and folded arms, should allow GEO 600 to reach a final sensitivity that is comparable to that of first generation gravitational-wave detectors with arm lengths of several kilometres (e.g. LIGO and VIRGO). In addition, dual recycling allows the photon shot noise limit of GEO 600 to be shaped. This can be seen in figure 1, which shows the expected noise spectral density of GEO 600 for two different modes of DRMI operation.

This paper will focus on the performance of GEO 600 during S3, a data-taking run performed in coincidence with LIGO and TAMA, and the first run during which GEO 600 was operated with dual recycling. It will also give a brief overview of the detector commissioning and characterization work relevant to this period and the remaining steps that are planned to bring GEO 600 into its final configuration.

\section{The dual-recycled Michelson interferometer}

\subsection{Layout of the dual-recycled Michelson interferometer}

A simplified optical layout of GEO 600 during S3 and as of March 2004 is shown in figure 2. A Nd:YAG laser system consisting of a high power 'slave' locked to a low power 'master' laser provides an output power of about $12 \mathrm{~W}$. Because photon shot noise is currently not a limiting noise source, and in order to avoid damage to components such as photodiodes during detector commissioning, the laser power is attenuated to $2 \mathrm{~W}$ before entering the first mode 

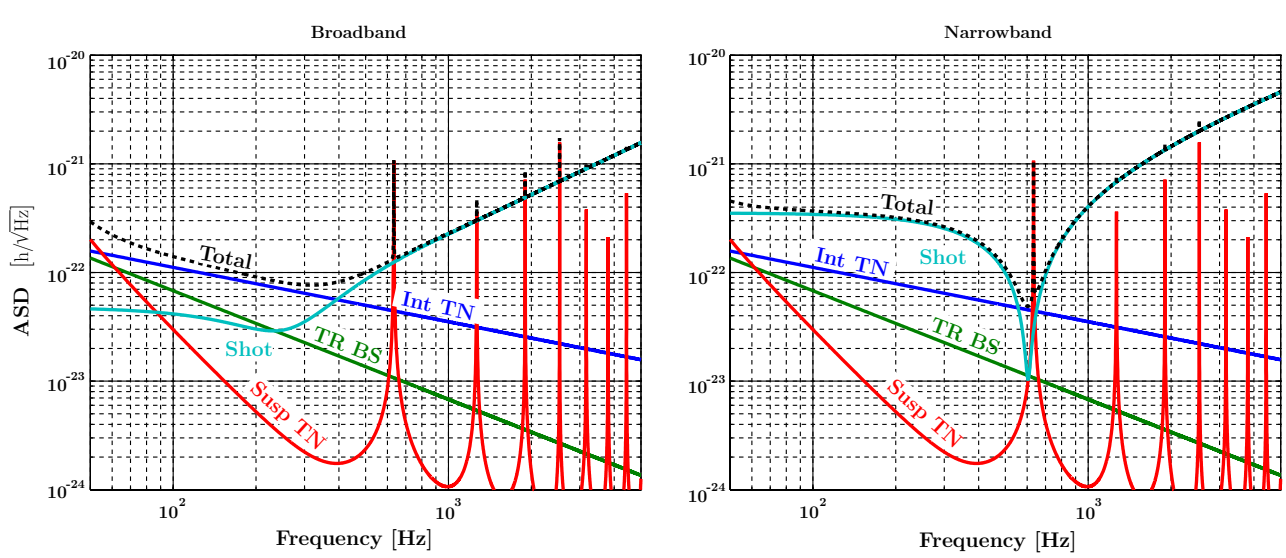

Figure 1. Expected noise spectral density of GEO 600 shown for two different modes of operation: broadband signal recycling on the left and narrowband signal recycling on the right. Contributions to the total noise curve come from internal thermal noise of the test mass substrates and coatings, thermorefractive noise of the beamsplitter, suspension thermal noise and shot noise. Seismic noise is expected to limit sensitivity below $50 \mathrm{~Hz}$.

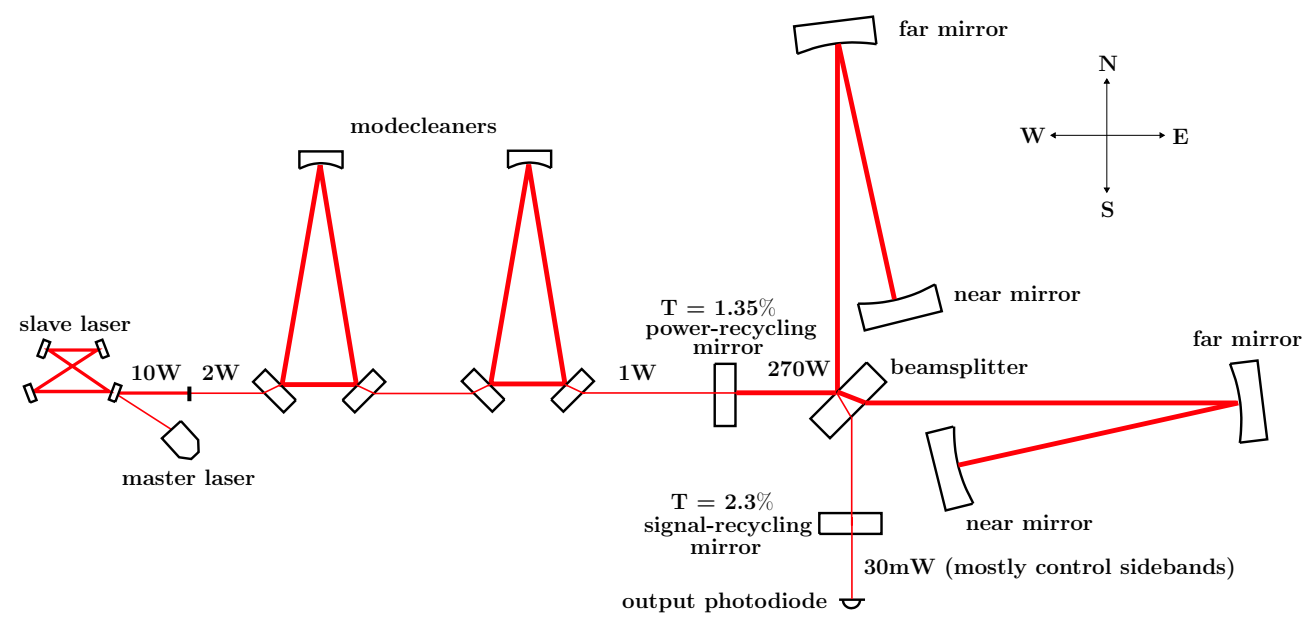

Figure 2. Simplified layout of the optical configuration of GEO 600 during S3 and as of March 2004. Approximate laser powers are given for various points within the sytem.

cleaner. The two mode cleaner cavities provide temporal and spatial filtering of the laser light and have a $50 \%$ throughput in series resulting in about $1 \mathrm{~W}$ incident on the power-recycling mirror.

The Michelson interferometer (MI) is formed by the beamsplitter and two (nearly) orthogonal arms, each folded once via the far mirrors to give an effective arm length of $1200 \mathrm{~m}$. Differential length changes between the arms of the MI create sidebands on the light, the so-called signal sidebands, which interfere at the beamsplitter and propagate southward towards the output port.

The power-recycling cavity (PRC) is formed by the power-recycling mirror and the MI. The laser frequency is kept resonant (by a servo) for the length of this cavity, resulting in an enhancement of the laser power within the PRC. The current power buildup, determined by the transmittance of the power-recycling mirror, $T \approx 1.35 \%$, is roughly $4 / T \approx 270$. 
The signal-recycling cavity (SRC) is formed by the signal-recycling mirror and the MI. The microscopic position of the signal-recycling mirror, and thus the common-mode length of this cavity, can be adjusted, or tuned, in order to provide resonant enhancement of signal sidebands in a band centred around a chosen Fourier frequency, the tuning frequency. The bandwidth of this cavity is about $750 \mathrm{~Hz}$, primarily determined by the transmittance of the signal-recycling mirror, $T \approx 2.3 \%$. Together, the PRC, SRC and MI form the dual-recycled Michelson interferometer (DRMI). For a more detailed description of the DRMI see [5].

The signal sidebands transmitted by the signal-recycling mirror are finally detected by the output photodiode. The output signal of this photodiode is then calibrated in order to produce a strain signal for gravitational-wave analysis. For a description of the calibration process see [13].

\subsection{Commissioning of the dual-recycled Michelson interferometer}

Obtaining signal-recycling cavity locks proved to be a major challenge during the commissioning of the DRMI. The error signal used for locking the length of the SRC is only valid over a microscopic range $(<1 \mathrm{~nm})$ of cavity length around its nominal operating point (see figure 2 in [5]). Thus, the limited force of the actuators used to adjust the cavity length (via the longitudinal position of the signal-recycling mirror) sets a limit on the speed of cavity length changes that can be tolerated of roughly $100 \mathrm{~nm} \mathrm{~s}^{-1}$. In addition, the validity of the error signal is greatly diminished by relatively small (order $100 \mathrm{nrad}$ ) misalignments of the mirrors that form the cavity. Early locking experiments showed that mirror motions during normal conditions permitted only a few short locks of the SRC per day.

Investigations into the cause of mirror motions revealed a larger than expected coupling factor for the main triple-pendulum suspensions between longitudinal motion (along the beam axis) of the upper and intermediate stages to tilt the motion of the mirrors. Under normal conditions, this coupling resulted in mirror tilt misalignments of a few $\mu \mathrm{rad} / \sqrt{\mathrm{Hz}}$ around $1 \mathrm{~Hz}$.

In order to reduce the resulting mirror misalignments, a bipartite digital feed-forward system was implemented [14]; in one part of this system, seismic motion along the beam axis of each mirror is monitored using Streckeisen STS-2 seismometers [15], and the signals are filtered and applied to piezoelectric crystals (PZTs) in the seismic-isolation stacks supporting the suspensions; in the other, the tilt introduced via longitudinal feedback to the intermediate masses of the near mirrors is compensated by a correction signal. This is generated from the longitudinal feedback, filtered and applied back to the tilt actuators on the intermediate masses.

In parallel with the implementation of the feed-forward system, a new locking scheme was developed, which allowed more robust lock acquisition of the DRMI. This scheme closely follows the one outlined in [5], but with the addition of a new initial lock of the SRC. Experiments and modelling showed that demodulating the signal from the output photodiode at double the modulation frequency used for the MI, while the PRC and MI are locked, produces a signal that represents the buildup of the sideband power within the SRC, which is largely determined by the position of the signal-recycling mirror. By subtracting an offset from this signal, a suitable error signal for locking the SRC at a large tuning frequency is produced. This signal is less sensitive to cavity misalignments, but also more noisy than the nominal error signal.

Using this so-called $2 f$ signal, the SRC is initially locked at a tuning of about $5.5 \mathrm{kHz}$, reducing the longitudinal motions of the signal-recycling mirror (and thus the length changes of the SRC). This allows the activation of the automatic alignment system [18], which reduces 

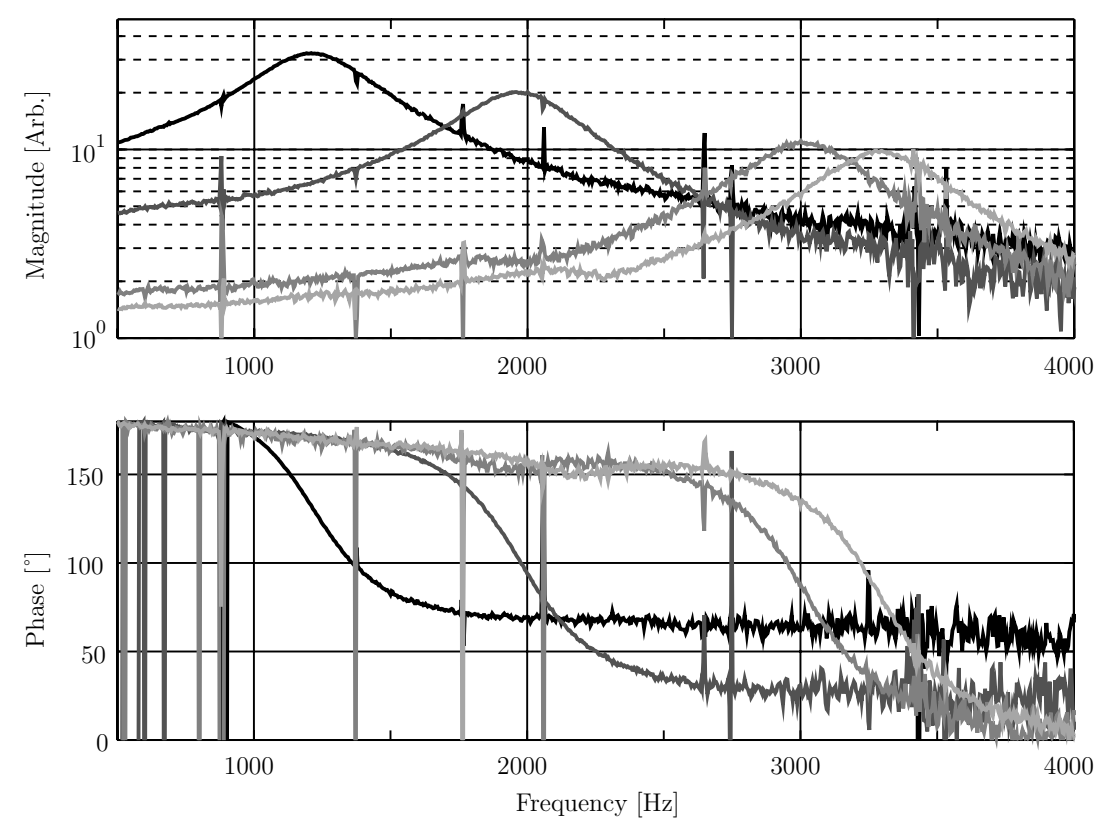

Figure 3. Transfer function (in arbitrary magnitude units) from a differential input to the electrostatic drive actuators behind the near mirrors to interferometer output for four different tuning frequencies. The transfer function of the actuators $\left(f^{-2}\right.$ for these frequencies) has been removed.

mirror misalignments in the DRMI, thereby improving the quality of the nominal signalrecycling error signal. Finally, the SRC control signal is switched from the $2 f$ signal to the nominal signal-recycling error signal by a microcontroller. This new locking scheme, combined with the reduction of mirror misalignment given by the feed-forward system, led to regular lock re-acquisition times on the order of minutes. The stability of the DRMI lock was also greatly improved. This was demonstrated during the S3 run, and is described in the following section.

Following the lock acquisition scheme outlined above, the SRC is initially locked to a tuning of about $5.5 \mathrm{kHz}$. However, it is desirable to tune to lower frequencies to allow for maximum signal enhancement within the gravitational-wave band (i.e. below a few $\mathrm{kHz}$ ). This is done automatically by adjusting the common-mode length of the SRC (and relevant parameters such as gains, modulation frequencies and phases) in a stepwise fashion using a computer program based on look-up tables. For more details on the principles of the downtuning process refer to [16]. During the S3 run, the final tuning frequency was set close to $1 \mathrm{kHz}$, where non-optical noise contributions had been minimized. From the time of a $2 f$-lock acquisition, this frequency could be reached in about $30 \mathrm{~s}$.

\subsection{Characterization of the dual-recycled Michelson interferometer}

For a DRMI, the transfer function from differential length changes of the arms of the MI to the interferometer output is not flat with frequency, as it is for a power-recycled Michelson interferometer (PRMI), but rather frequency-dependent due to the resonant enhancement of signal sidebands within the SRC. This effect can be seen in figure 3. This tunable optical gain gives GEO 600 the ability, in principle, to run targeted searches for known astrophysical sources, or to follow sources that move in frequency. 


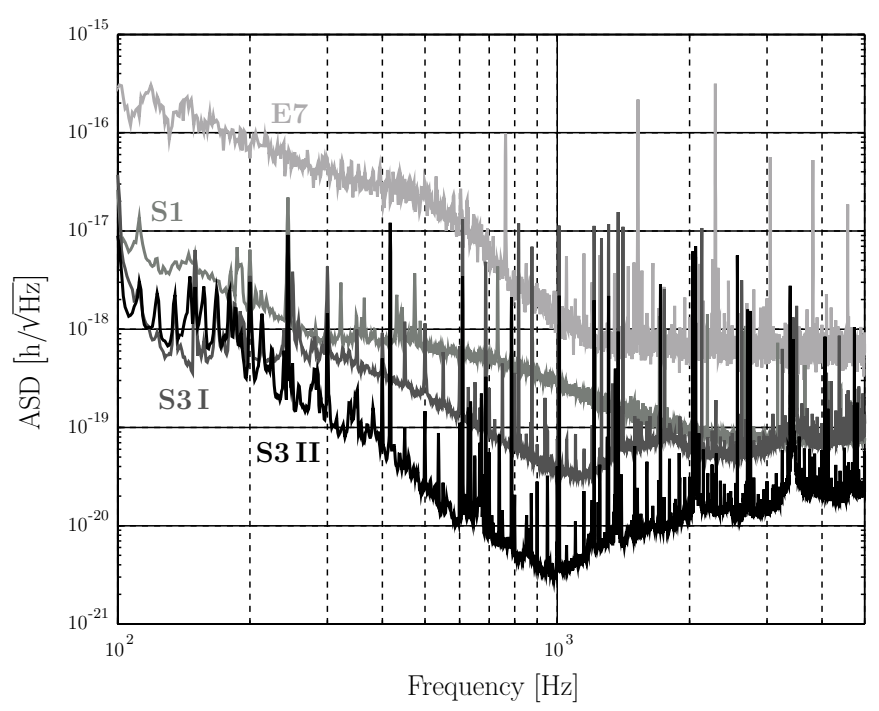

Figure 4. Typical sensitivity of GEO 600 during the E7 engineering run, S1 science run, and S3 I and S3 II periods of the S3 science run.

\section{Detector performance during the $\mathbf{S 3}$ science run}

The third LIGO Scientific Collaboration (LSC) science run, S3, was performed between 1 November 2003 and 9 January 2004. The commissioning work described above produced a stably-locking DRMI only about two weeks before the start of S3, and thus left little time for testing and sensitivity optimization. Therefore it was decided that GEO 600 would run for about one week, near the beginning of S3, then go offline to allow time to improve the sensitivity and stability of the detector, and finally rejoin the run.

Thus, the GEO 600 S3 science run was divided into two separate periods of time, having somewhat different interferometer configurations and sensitivities. The first ran from 5 November through 11 November, and the second from 30 December to 13 January (a few days after the end of the LSC S3). We refer to these as 'S3 I' and 'S3 II' respectively, and ' $\mathrm{S} 3$ ' is used when both periods are relevant. We refer to the 47 day period between S3 I and S3 II as the 'engineering period'. The following subsections give an overview of the status of GEO 600 during S3 I and S3 II, as well as a description of the work undertaken during the engineering period.

\subsection{The S3 I data run}

During S3 I, GEO 600 was operated in the DRMI configuration described in section 2 with the SRC tuning frequency set close to $1.3 \mathrm{kHz}$. The improvement in sensitivity for this run, compared to the previous runs, during which GEO 600 was operated as a PRMI (i.e. without signal recycling), can be seen in figure 4.

The detector was monitored by at least one (remote) operator at all times during S3 I, although human interaction was rarely required. Unlike in previous runs, there were no scheduled maintenance periods during S3 I or S3 II. Instead, if there was a need to enter one of the main buildings (e.g. to adjust parameters that could not be accessed remotely), an operator could flip a switch upon entrance and exit from the building, creating a signal that was recorded by the data acquisition system [17], thus defining the duration of the maintenance. 
Table 1. Duty cycle statistics for S3 I. Here 'days' are defined as $24 \mathrm{~h}$ periods starting on 5 November 2003, 13:30:00 UTC, the start time of S3 I, with the exception of day 7, which encompasses $25.5 \mathrm{~h}$ due to the fact that S3 I lasted for 169.5 hours.

\begin{tabular}{lcc}
\hline Day & Duty cycle $(\%)$ & Losses of lock \\
\hline 1 & 100 & 0 \\
2 & 99.2 & 3 \\
3 & 98.1 & 1 \\
4 & 99.5 & 1 \\
5 & 93.6 & 1 \\
6 & 98.8 & 2 \\
7 & 77.9 & 8 \\
Total & 95.2 & 16 \\
\hline
\end{tabular}

Table 2. Causes of loss of lock during S3 I.

\begin{tabular}{lc}
\hline Cause of loss of lock & Number \\
\hline North far mirror suspension temperature & 8 \\
Seismic event & 2 \\
Signal-recycling mirror feedback saturation & 1 \\
Mode cleaner alignment jumps & 1 \\
Alignment feedback saturation & 1 \\
Corrupted frame data file & 1 \\
Unidentified & 2 \\
Total & 16 \\
\hline
\end{tabular}

The DRMI showed remarkable lock stability during S3 I, with a duty cycle of better than $95 \%$. Some daily lock statistics for this period are shown in table 1. These statistics were generated by defining a 'locked second' as any GPS second for which there was not a maintenance period, and time-domain calibrated detector output, $h(t)$, was produced. For S3 I, $h(t)$ production began about $30 \mathrm{~s}$ after the DRMI was locked and the SRC had reached the final detuning frequency, and stopped about $24 \mathrm{~s}$ before each loss of interferometer lock (the data were post-processed). Thus, a 'loss of lock' here is any event that causes an interruption of the sequence of locked seconds (which is not always an unlocked interferometer).

Investigation of recorded signals for the times before each loss of lock yielded six different causes, with two losses of lock left unidentified. The results are presented in table 2. A decrease in the temperature within the north end building on the last day of S3 I caused the most losses of lock, 8 of the total 16 . This was due to a mechanical contact (likely between the upper mass and the coil holder) on the north far mirror triple-pendulum suspension.

\subsection{Engineering period}

The period following S3 I was used to improve the lock stability and sensitivity of the detector. The former was achieved by addressing the causes of loss of lock during S3 I that have been presented in table 2. To reduce the likelihood of unwanted mechanical contacts involving the north far mirror suspension, an electric radiative heater with a thermostat was installed close to the vacuum tank enclosing the suspension. To avoid saturation of the feedback to the signal-recycling mirror, a slow-drift control, similar to those already implemented for many other degrees of freedom within the automatic alignment system [18], was implemented for the SRC. Finally, a bad contact was found within the circuitry of the automatic alignment system for the mode cleaners, and bypassed. 
Table 3. Duty cycle statistics for S3 II. Here 'days' are defined as $24 \mathrm{~h}$ periods starting on 30 December 2003, 15:00:00 UTC, the start time of S3 II, with the exception of day 14, which is actually $25 \mathrm{~h}$ long due to the fact that S3 II lasted for $337 \mathrm{~h}$.

\begin{tabular}{lcc}
\hline Day(s) & Duty cycle $(\%)$ & Losses of lock \\
\hline $1-2$ & 100 & 0 \\
3 & 93.8 & 9 \\
4 & 100 & 0 \\
5 & 92.6 & 4 \\
6 & 99.9 & 1 \\
7 & 99.7 & 1 \\
$8-9$ & 100 & 0 \\
10 & 99.7 & 1 \\
$11-13$ & 100 & 0 \\
14 & 95.9 & 3 \\
Total & 98.7 & 19 \\
\hline
\end{tabular}

Investigations made during this period indicated that the limiting noise sources during S3 I were: noise introduced by the servo used to control the longitudinal degree of freedom of the SRC; electronic noise of the high power photodiode used to measure the out-of-loop differential arm length error-point of the MI; and phase noise of the function generator that supplied the modulation/demodulation frequency signal for the frontal modulation locking of the differential arm length of the MI. The noise level introduced by the SRC servo was reduced by changing the gain distribution within the servo electronics. The MI function generator and high power photodiode were replaced with models with lower phase and electronic noise, respectively. These changes led to the improved sensitivity during S3 II, which can be seen in figure 4.

\subsection{The S3 II data run}

The configuration of GEO 600 during S3 II was basically the same as that of S3 I, except for the changes discussed above. In addition, the SRC tuning frequency was reduced to $1 \mathrm{kHz}$. The influence of these changes on the sensitivity can be seen in figure 4 .

Unlike in S3 I, during S3 II the detector was not continuously monitored by operators. While at least one operator was on site during normal working hours, much of the run was conducted without any operators on site, or even awake. For the times when operators were not on site, a text messaging alarm system was used. When certain parameters were out of range, the system sent informative messages to the mobile phone of an operator on duty. Having received such a message, the operator could check the situation remotely via the internet, and react in a relatively short time, if deemed necessary.

The changes described in the last section led to an increase in the stability of the interferometer for S3 II. The overall duty cycle of this run was over $98 \%$, with 5 locks lasting longer than $24 \mathrm{~h}$, and one longer than $95 \mathrm{~h}$. Some daily lock statistics for this run are given in table 3. These statistics were produced using the same method used for S3 I. A comprehensive investigation into the causes of loss of lock for S3 II has not yet been done.

\section{Future plans}

After the completion of S3, an investigation of technical noise contributions to the detector output was undertaken, leading to the identification of several noise sources that were directly 
limiting the sensitivity. One of these was the electronic noise of the quadrant photodiode that was used to produce the error signal used for MI control. Switching the control input to the error signal generated from the output of the less-noisy high power photodiode- - which was already being used to measure the out-of-loop MI error signal-resulted in a reduction of the noise in the detector output between 50 and $500 \mathrm{~Hz}$ by as much as a factor of 10 . Further investigation and elimination of technical noise are expected to comprise much of the work in the near future.

In addition to this, several major steps remain before GEO 600 will reach its final configuration. In order to target astrophysical sources, it is desirable to be able to tune the resonance of the SRC to all frequencies within the GEO 600 detection band $(50-6000 \mathrm{~Hz})$. Therefore, the down-tuning process must be extended. Recent experiments have shown successful down-tuning to $550 \mathrm{~Hz}$, and there is no reason, in principle, that this cannot be extended to even lower tuning frequencies. In order to decrease the relative photon shot-noise of the detected light to a level that will allow GEO 600 to reach its design sensitivity it will be necessary to increase the laser power within the interferometer. Thus an increase to the full injected laser power is planned for the coming months. After this, in order to facilitate the final power within the PRC, the current power-recycling mirror will be replaced with a more highly reflective mirror.

\section{Acknowledgments}

We would like to thank PPARC in the UK and the BMBF and the state of Lower Saxony, Germany.

\section{References}

[1] Schutz B F 1999 Class. Quantum Grav. 16 A131

[2] Sigg D et al 2004 Class. Quantum Grav. 21 S409

[3] Takahashi R et al 2004 Class. Quantum Grav. 21 S403

[4] Frasconi F et al 2004 Class. Quantum Grav. 21 S385

[5] Grote H, Freise A, Malec M, Heinzel G, Willke B, Lück H, Strain K A, Hough J and Danzmann K 2004 Class. Quantum Grav. 21 S473

[6] Meers B J 1988 Phys. Rev. D 382317

[7] Strain K A and Meers B J 1991 Phys. Rev. Lett. 661391

[8] Heinzel G et al 1998 Phys. Rev. Lett. 815493

[9] Goßler S, Cagnoli G, Crooks D R M, Lück H, Rowan S, Smith J R, Strain K A, Hough J and Danzmann K 2004 Class. Quantum Grav. 21 S923

[10] Smith J R, Cagnoli G, Crooks D R M, Fejer M M, Goßler S, Lück H, Rowan S, Hough J and Danzmann K 2004 Class. Quantum Grav. 21 S1091

[11] Lück H, Freise A, Goßler S, Hild S, Kawabe K and Danzmann K 2004 Class. Quantum Grav. 21 S985

[12] Goßler S et al 2003 Rev. Sci. Instrum. 743787

[13] Hewitson M et al 2004 Class. Quantum Grav. 21 S1711

[14] Smith J R et al in preparation

[15] Streckeisen G AG Messgeräte Dättlikonerstrasse 5, CH-8422 Pfungen Switzerland

[16] Malec M, Grote H, Freise A, Heinzel G, Strain K A, Hough J and Danzmann K 2004 Class. Quantum Grav. 21 S991

[17] Kötter K et al 2002 Class. Quantum Grav. 191399

[18] Grote H, Heinzel G, Freise A, Goßsler S, Willke B, Lück H, Ward H, Casey M M, Strain K A, Robertson D I, Hough J and Danzmann K 2004 Class. Quantum Grav. 21 S441 